This is the accepted manuscript made available via CHORUS. The article has been published as:

\title{
Suppression of exciton-exciton annihilation in tungsten disulfide monolayers encapsulated by hexagonal boron nitrides
}

Yusuke Hoshi, Takashi Kuroda, Mitsuhiro Okada, Rai Moriya, Satoru Masubuchi, Kenji Watanabe, Takashi Taniguchi, Ryo Kitaura, and Tomoki Machida

Phys. Rev. B 95, 241403 - Published 7 June 2017

DOI: 10.1103/PhysRevB.95.241403 
1 Suppression of exciton-exciton annihilation in tungsten disulfide 2 monolayers encapsulated by hexagonal boron nitrides

3 Yusuke Hoshi ${ }^{*}$, , Takashi Kuroda ${ }^{2}$, Mitsuhiro Okada ${ }^{3}$, Rai Moriya ${ }^{1}$, Satoru Masubuchi ${ }^{1}$,

4 Kenji Watanabe ${ }^{2}$, Takashi Taniguchi ${ }^{2}$, Ryo Kitaura ${ }^{3}$, and Tomoki Machida ${ }^{\dagger, 1}$

6 Institute of Industrial Science, University of Tokyo, 4-6-1 Komaba, Meguro-ku, Tokyo

7 153-8505, Japan

$8{ }^{2}$ National Institute for Materials Science, 1-1 Namiki, Tsukuba, Ibaraki 305-0044, 9 Japan

$10{ }^{3}$ Department of Chemistry, Nagoya University, Furocho, Chikusa-ku, Nagoya 464-8603,

11 Japan

Corresponding author. Email:

*yuhoshi@iis.u-tokyo.ac.jp (Y. Hoshi),

$15{ }^{\dagger}$ tmachida@iis.u-tokyo.ac.jp (T. Machida).

\section{ABSTRACT}

We investigates exciton-exciton annihilation (EEA) in tungsten disulfide $\left(\mathrm{WS}_{2}\right)$ monolayers encapsulated by hexagonal boron nitride $(\mathrm{hBN})$. It is revealed that decay

21 signals observed by time-resolved photoluminescence (PL) are not strongly dependent on the exciton densities of $\mathrm{hBN}$-encapsulated $\mathrm{WS}_{2}$ monolayers $\left(\mathrm{WS}_{2} / \mathrm{hBN}\right)$. In contrast, the sample without the bottom $\mathrm{hBN}$ layer $\left(\mathrm{WS}_{2} / \mathrm{SiO}_{2}\right)$ exhibits a drastic decrease of decay time with increasing exciton density due to the appearance of a rapid PL decay component, signifying nonradiative EEA-mediated recombination. Furthermore, the

26 EEA rate constant of $\mathrm{WS}_{2} / \mathrm{hBN}$ was determined as $(6.3 \pm 1.7) \times 10^{-3} \mathrm{~cm}^{2} \mathrm{~s}^{-1}$, being about two orders of magnitude smaller than that of $\mathrm{WS}_{2} / \mathrm{SiO}_{2}$. Thus, the observed EEA rate reduction played a key role in enhancing luminescence intensity at high exciton

29 densities in the $\mathrm{WS}_{2}$ monolayer. 

platform for realizing an ideal, atomically thin, and optically accessible two-dimensional system. In particular, TMDs of $\mathrm{MX}_{2}$ stoichiometry $(\mathrm{M}=\mathrm{Mo}, \mathrm{W} ; \mathrm{X}=\mathrm{S}$,

Se, Te) are considered promising materials for fundamental physics research, exhibiting unique properties such as direct bandgaps of single monolayers (1L) and extraordinary large binding energies. ${ }^{1-4}$ These properties lead to exciton formation even at room temperature, with their recombination dynamics being important for TMD applications in optoelectronic devices. ${ }^{5-11}$ The optical properties of $1 \mathrm{~L}-\mathrm{TMDs}$ are quite sensitive to the nature of supporting substrates ${ }^{12}$ and other environmental conditions, ${ }^{13,14}$ which induce changes of background doping levels and the exciton recombination rate.

The enhanced many-body effects arising from strong interactions between excitons at high exciton density are another prominent feature of low-dimensional systems. Among the numerous processes of multiexciton dynamics, involving species such as charged excitons, ${ }^{15,16}$ biexcitons, ${ }^{17,18}$ and exciton-trion complexes, ${ }^{19}$ exciton-exciton annihilation (EEA) is one of the most extensively studied ones. ${ }^{20-29}$ EEA is a scattering mechanism in which two excitons come into contact, with one of them undergoing nonradiative recombination and transferring its energy to another exciton that is then excited to a high-energy continuum state. Subsequently, the excited exciton undergoes thermal relaxation without emitting light. This process is identical to the Auger relaxation of inner-shell electrons following the photoionization of atoms, being a source of luminescence quenching. EEA occurs at ultrafast time scales of several picoseconds, limiting the radiative decay of excitons under intense photoexcitation. Thus, EEA suppression is critical for enhancing the performance of optoelectronic devices. The recently reported EEA rate constants of suspended TMDs are of the order of $\sim 10^{-1} \mathrm{~cm}^{2} \mathrm{~s}^{-1}$, giving rise to EEA at a markedly small exciton density 
of $\sim 10^{10} \mathrm{~cm}^{-2} \cdot{ }^{24,26}$ In contrast, the corresponding rate constants of TMDs supported by

$57 \mathrm{SiO}_{2}$ films are smaller, ${ }^{23,25,26}$ still being about two orders of magnitude larger than those of conventional two-dimensional quantum wells such as $\mathrm{ZnCdSe} / \mathrm{ZnSe}$ heterostructure. $^{30}$ Interactions of excitons with their surroundings may suppress nonradiative EEA-mediated recombination in TMDs. Hexagonal boron nitride $(\mathrm{hBN})$ is a prominent atomically flat dielectric surrounding material for layered structures such as graphene and TMDs reducing their surface roughness and charged impurity scattering

63 of carriers. ${ }^{31,32}$ Thus, encapsulation of layered materials by hBN is advantageous not only for device-related applications but also for investigating the fundamental physics of these materials. TMDs encapsulated by $\mathrm{hBN}$ have been reported to exhibit significantly narrow spectral lines with reduced inhomogeneous broadening. ${ }^{33}$ In this study, we focus on the behavior of excitons in the EEA process occurring in $1 \mathrm{~L}-\mathrm{WS}_{2}$ encapsulated by $\mathrm{hBN}\left(\mathrm{hBN} / \mathrm{WS}_{2}\right)$, with $\mathrm{WS}_{2}$ chosen as a model material due to its low defect density and a high PL yield at room temperature, as compared to those of other 10-30 nm thick $\mathrm{W}$ layer on a sapphire substrate by sputtering, thermal oxidation at $700{ }^{\circ} \mathrm{C}$, and sulfurization at $900{ }^{\circ} \mathrm{C} .{ }^{36}$ Bulk hBN crystals were grown employing a temperature-gradient method at high pressure $(4.0$ - $5.5 \mathrm{GPa})$ and high temperature $\left(1,500-1,750{ }^{\circ} \mathrm{C}\right) \cdot{ }^{37}$ Flakes of $\mathrm{hBN}$ were prepared by micromechanical exfoliation of 
$\mathrm{n}^{+}$-doped $\mathrm{Si}(100)$ using the stamping technique through a dry peel/lift process. The first sample comprised $1 \mathrm{~L}-\mathrm{WS}_{2}$ encapsulated by $\mathrm{hBN}$, with the top-to-bottom layer sequence being $\mathrm{hBN} / 1 \mathrm{~L}-\mathrm{WS}_{2} / \mathrm{hBN} / \mathrm{SiO}_{2}$ (Fig. 1(a)). The second sample, serving as a reference, comprised hBN/1L-WS 2 directly formed on $\mathrm{SiO}_{2}$ without the bottom hBN layer (Fig. 1(b)). These two samples are further denoted as $\mathrm{WS}_{2} / \mathrm{hBN}$ (hBN-encapsulated) and $\mathrm{WS}_{2} / \mathrm{SiO}_{2}\left(\mathrm{SiO}_{2}\right.$-supported).

A standard confocal microscope with a focusing diameter of $\sim 1 \mu \mathrm{m}$ was used to observe the luminescence of $1 \mathrm{~L}-\mathrm{WS}_{2}$. Steady-state photoluminescence (PL) measurements were carried out using a continuous wave excitation laser emitting at 532 $\mathrm{nm}$ and a spectrometer equipped with a cooled charge-coupled device detector. Time-resolved PL (TRPL) measurements were carried out using an optical parametric oscillator generating picosecond pulses with a wavelength of $550 \mathrm{~nm}$ and a repetition rate of $76 \mathrm{MHz}$. PL decay curves were analyzed by a synchronously scanned streak camera with a minimum temporal resolution of $2 \mathrm{ps.} \mathrm{All} \mathrm{measurements} \mathrm{were} \mathrm{performed}$ at room temperature and ambient pressure.

Figures 1(a) and 1(b) show optical microscopy images of $\mathrm{WS}_{2} / \mathrm{hBN}$ and $\mathrm{WS}_{2} / \mathrm{SiO}_{2}$ samples, respectively, and Fig. 1(c) shows the steady-state PL spectra of each sample at low excitation power $\left(2.4 \mathrm{~kW} \mathrm{~cm}^{-2}\right)$. The above samples exhibit similar spectra, featuring an intense peak at $\sim 1.99 \mathrm{eV}$ and a small side peak at $1.95 \mathrm{eV}$, attributed to neutral excitons (X) and negative trions (T), respectively. The full width at half maximum (FWHM) of the exciton peak of $\mathrm{WS}_{2} / \mathrm{hBN}$ and $\mathrm{WS}_{2} / \mathrm{SiO}_{2}$ is 21 and 29 $\mathrm{meV}$, respectively. These values are comparable to that of $1 \mathrm{~L}-\mathrm{WS}_{2}$ directly grown on an $\mathrm{hBN}$ template, ${ }^{38}$ indicating that no crystal damage is inflicted during heterostructure fabrication. Figure 1(e) shows the dependence of exciton peak intensity on excitation power, revealing that $\mathrm{WS}_{2} / \mathrm{SiO}_{2}$ exhibits a sublinear dependence in the entire power 
106

107

108

109

110

111

112

113

range, with intensity saturation occurring at excitation powers around $100 \mathrm{~kW} \mathrm{~cm}$. This behavior is typical of power-induced nonradiative recombination, as confirmed for other TMD systems..$^{23,28,39,40}$ In contrast, the intensities exhibited by $\mathrm{WS}_{2} / \mathrm{hBN}$ are strictly proportional to excitation power up to $\sim 100 \mathrm{~kW} \mathrm{~cm}{ }^{-2}$, which marks the onset of saturation. The observed luminescence robustness with respect to input power is specific to the hBN-encapsulated sample. Consequently, at sufficiently high excitation power (e.g., $240 \mathrm{~kW} \mathrm{~cm}{ }^{-2}$ ), the PL intensity of $\mathrm{WS}_{2} / \mathrm{hBN}$ is an order of magnitude higher than that of $\mathrm{WS}_{2} / \mathrm{SiO}_{2}$ (Fig. 1(d)).

To demonstrate that encapsulation by hBN strongly affects exciton dynamics in $1 \mathrm{~L}-\mathrm{WS}_{2}$, we systematically investigated neutral exciton decay time by TRPL measurements. Figure 2(a) shows PL decay signals of $\mathrm{WS}_{2} / \mathrm{hBN}$ normalized with respect to initial exciton densities. Density estimation was performed by calculating the photon flux per pulse, and 1L-WS 2 was assumed to exhibit a linear absorbance of $3.5 \%$ for simplicity, independent of the substrate choice. ${ }^{41}$ Decay signals observed at initial exciton densities below $2.6 \times 10^{11} \mathrm{~cm}^{-2}$ are almost the same, being well described by a monoexponential curve with a time constant $\tau_{\mathrm{hBN}}$ of $\sim 60 \mathrm{ps}$. At a high exciton density of $2.6 \times 10^{12} \mathrm{~cm}^{-2}$, the data can no longer be fitted by a monoexponential curve due to the

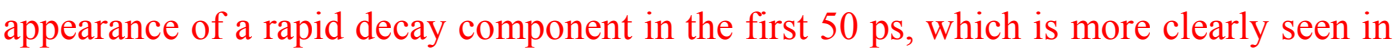
Fig. 3(d), where the experimental data is plotted in a linear scale axis without the normalization. Figure 2(b) shows the exciton PL decay signals of $\mathrm{WS}_{2} / \mathrm{SiO}_{2}$, with that for the lowest exciton density $\left(2.6 \times 10^{10} \mathrm{~cm}^{-2}\right)$ described by a monoexponential curve with a decay time $\tau_{\mathrm{SiO} 2}$ of 590 ps. Significantly faster decay is observed for a ten-fold higher exciton density $\left(2.6 \times 10^{11} \mathrm{~cm}^{-2}\right)$, with the average decay time decreasing to 46 ps. Herein, we utilized the average decay time as an alternative to the decay time, since the measured signal deviated from monoexponential decay behavior. The average decay 
131

132

$149 \quad \frac{d n}{d t}=-\frac{n}{\tau}-\gamma n^{2}$ where $\gamma$ is the EEA rate constant, and $\tau$ is the excitonic decay time in the absence of EEA. ${ }^{25,27,43}$ For a time-independent annihilation rate constant $\gamma$, the linear solution of Eqn. (1) can be represented by

$$
\frac{1}{n}=A e^{t / \tau}-\gamma \tau,
$$

154 where $A$ equals $\left(1 / n_{0}+\gamma \tau\right)$, and $n_{0}$ is the initial exciton density. The intensity-dependent 
data for $\mathrm{WS}_{2} / \mathrm{hBN}$ and $\mathrm{WS}_{2} / \mathrm{SiO}_{2}$ are replotted in the linear form given by Eqn. (2) (Figs. 3(a) and 3(b)), with $A$ determined as the slope of the corresponding linear fit including the error. Figure 3(c) shows a plot of $A$ as a function of $1 / n_{0}$ for both samples. The above results are successfully fitted by a linear function with a tilt of one. EEA rate constants $\gamma$ are estimated from the values of $A$ at $1 / n_{0}=0$. The estimated EEA rate constants are given in Table I. Notably, the $\gamma$ value of $\mathrm{WS}_{2} / \mathrm{hBN}\left((6.3 \pm 1.7) \times 10^{-3} \mathrm{~cm}^{2}\right.$ $\left.\mathrm{s}^{-1}\right)$ is much smaller than that of $\mathrm{WS}_{2} / \mathrm{SiO}_{2}\left((1.2 \pm 0.1) \times 10^{-1} \mathrm{~cm}^{2} \mathrm{~s}^{-1}\right)$ which, in turn, is very similar to the rate constant $\left(\sim 10^{-1} \mathrm{~cm}^{2} \mathrm{~s}^{-1}\right)$ of $1 \mathrm{~L}-\mathrm{WS}_{2}$ directly deposited on $\mathrm{SiO}_{2}{ }^{25,26}$ The error bars of the rate constant are brought in the linear fitting for inducing the slope $A$. To verify the validity of the obtained EEA rate constants, they are used to reproduce the decay curves using Eqn. (2), achieving good agreement with experimental results for both $\mathrm{WS}_{2} / \mathrm{hBN}$ and $\mathrm{WS}_{2} / \mathrm{SiO}_{2}$ (Figs. 3(d) and 3(e), respectively). In contrast, the decay curves calculated from the above rate equation without the EEA term $\left(n=n_{0} \times\right.$ $\exp (t / \tau))$ obviously deviate from the experimental results for both samples. Thus, the reduction of decay time dependence on initial exciton density is caused by the EEA-mediated optical transition, with the rate constant of $\mathrm{WS}_{2} / \mathrm{hBN}$ being about two orders of magnitude smaller than that of $\mathrm{WS}_{2} / \mathrm{SiO}_{2}$. This result strongly indicates that encapsulation by $\mathrm{hBN}$ allows the EEA-mediated nonradiative recombination in $1 \mathrm{~L}-\mathrm{WS}_{2}$ to be suppressed.

In general, the EEA rate constant is known to be proportional to the exciton diffusion constant $D$ and the separation $R$ of two excitons at the point of EEA occurrence. ${ }^{26,43}$ At first, we discuss the effect of $D$ on the EEA rate constant of $\mathrm{WS}_{2} / \mathrm{hBN}$. Considering the mechanisms of carrier mobility enhancement in graphene encapsulated by $\mathrm{hBN},{ }^{44}$ the value of $D$ for $\mathrm{WS}_{2} / \mathrm{hBN}$ should exceed that of $\mathrm{WS}_{2} / \mathrm{SiO}_{2}$. Thus, the exciton diffusion coefficient is not related to the extraordinarily small EEA 
rate constant of $\mathrm{WS}_{2} / \mathrm{hBN}$. On the other hand, encapsulation by $\mathrm{hBN}$ decreases the

181 exciton binding energy because of the change in the dielectric environment ${ }^{14,45}$. The 182 encapsulation could subsequently increase $R$ since the exciton Bohr radius increases 183 with decreasing exciton binding energy ${ }^{46}$, accelerating the EEA. Thus, the separation $R$ 184 of two excitons cannot also explain the extraordinarily small EEA rate constant of $185 \mathrm{WS}_{2} / \mathrm{hBN}$. To rationalize the abovementioned EEA rate constant, we proposed a model 186 based on uniform exciton dispersion in the $2 \mathrm{D}$ plane. For $1 \mathrm{~L}-\mathrm{WS}_{2}$ on $\mathrm{SiO}_{2}$, excitons 187 may be localized due to potential fluctuation in $1 \mathrm{~L}-\mathrm{WS}_{2}$ owing to the roughness of the $1881 \mathrm{~L}-\mathrm{WS}_{2} / \mathrm{SiO}_{2}$ interface and the fixed charges in the vicinity of the $\mathrm{SiO}_{2}$ surface. ${ }^{47}$ In this 189 case, the localized exciton density should be larger than the injected exciton density due 190 to exciton collection in the potential minima of $1 \mathrm{~L}-\mathrm{WS}_{2}$. Therefore, EEA-mediated 191 nonradiative recombination in $\mathrm{WS}_{2} / \mathrm{SiO}_{2}$ probably occurs at a lower injected exciton density. On the other hand, encapsulation by hBN enables the generation of delocalized excitons in $1 \mathrm{~L}-\mathrm{WS}_{2}$ due to the smaller interface roughness and the separation from charged impurities in $\mathrm{SiO}_{2}$. In fact, the faster PL signal decay observed at low exciton densities (Fig. 2(c)) suggests the existence of delocalized excitons in $\mathrm{WS}_{2} / \mathrm{hBN}$. When uniformly dispersed in the 2D plane, these excitons are easily captured at crystal defects, explaining the quick PL signal decay of low-density delocalized excitons. ${ }^{48}$ Additionally, we measured steady-state PL spectra of these samples under cryogenic conditions (around $4 \mathrm{~K}$ ), and found that strong bound exciton emissions are clearly observed only for the $\mathrm{WS}_{2} / \mathrm{SiO}_{2}$ sample (not shown). For $\mathrm{WS}_{2} / \mathrm{hBN}$, exciton delocalization should contribute to the extraordinary small rate constant of EEA, which occurs upon contact of two excitons.

As a guide for further luminescence yield enhancement at high exciton densities, we calculated the time-integrated form $\int n(t) d t$ of exciton density derived 
from Eqn. (2) (see the Supplemental Material [42] for the dependence of the

206

207 time-integrated exciton density on the annihilation rate constant), which should correspond to the luminescence intensity generated by $1 \mathrm{~L}-\mathrm{WS}_{2}$. For samples with large EEA rate constants, the exciton PL decay time does not strongly affect the time-integration of exciton density. In contrast, for samples with smaller EEA rate constants, such as $\mathrm{WS}_{2} / \mathrm{hBN}$, an increase in PL decay time can enhance luminescence intensity. The exciton PL decay time $\tau$ is related to the radiative decay time $\tau_{r}$ and the nonradiative decay time $\tau_{n r}$ of excitons: $1 / \tau=1 / \tau_{r}+1 / \tau_{n r}$, with $\tau_{r}$ predicted to be of the order of several nanoseconds. ${ }^{49}$ Therefore, reducing the number of nonradiative recombination centers such as $\mathrm{S}$ vacancies should drastically enhance the luminescence intensity of samples with smaller EEA rate constants. For example, the quantum yield of $1 \mathrm{~L}-\mathrm{WS}_{2}$ has recently been reported to reach a value close to unity after treatment with a nonoxidizing organic superacid. ${ }^{50}$ Thus, larger luminescence yields might be realized by suppressing nonradiative pathways involving crystal defects in hBN-encapsulated 1L-WS .

In conclusion, we systematically investigated the effect of encapsulation by $\mathrm{hBN}$ on the exciton transition through the EEA in the $1 \mathrm{~L}-\mathrm{WS}_{2}$, which takes place at the high exciton density. It was found that the luminescence robustness with respect to input power was specific to the hBN-encapsulated sample. We quantitatively evaluated the EEA rate constant of the $\mathrm{hBN}$-encapsulated $1 \mathrm{~L}-\mathrm{WS}_{2}$ by using time-resolved PL measurements, and demonstrated that the EEA rate constant of the $\mathrm{WS}_{2} / \mathrm{hBN}$ was about two orders of magnitude smaller than that of $\mathrm{WS}_{2} / \mathrm{SiO}_{2}$, indicating that the $\mathrm{hBN}$-encapsulation results in high quantum yields at high exciton density in $1 \mathrm{~L}-\mathrm{WS}_{2}$. Earlier experiments on $1 \mathrm{~L}-\mathrm{MoS}_{2}{ }^{33}$ suggested the encapsulation by $\mathrm{hBN}$ layers made it possible to access its intrinsic high optical quality owing to the surface protection and 
substrate flatness. Also for $1 \mathrm{~L}-\mathrm{WS}_{2}$ in the present study, the $\mathrm{hBN}$ encapsulation revealed the extraordinarily small EEA rate constant. Thus, the encapsulation by $\mathrm{hBN}$ could be the rational design for wide variety of TMD materials in optoelectronics and fundamental physics.

\section{ACKNOWLEDGEMENTS}

This work was partly supported by the Japan Science and Technology Agency (JST) the Core Research for Evolutional Science and Technology (CREST) Grant Number JPMJCR15F3; JSPS KAKENHI Grant Numbers JP25107003, JP25107004, JP15K21722, JP26248061, JP25107002, and JP16H00982; and the Sasagawa Scientific Research Grant from The Japan Science Society.

241

242 


\section{REFERENCES}

244 [1] K. F. Mak, K. He, C. Lee, G. H. Lee, J. Hone, T. F. Heinz, and J. Shan, Nat. Mater. $245 \quad \mathbf{1 2}, 207(2013)$.

246 [2] A. Chernikov, T. C. Berkelbach, H. M. Hill, A. Rigosi, Y. Li, O. B. Aslan, D. R. 247 Reichman, M. S. Hybertsen, and T. F. Heinz, Phys. Rev. Lett. 113, 076802 (2014).

248 [3] K. He, N. Kumar, L. Zhao, Z. Wang, K. F. Mak, H. Zhao, and J. Shan, Phys. Rev. 249 Lett. 113, 026803 (2014).

250 [4] H. M. Hill, A. F. Rigosi, C. Roquelet, A. Chernikov, T. C. Berkelbach, D. R. 251 Reichman, M. S. Hybertsen, L. E. Brus, and T. F. Heinz, Nano Lett. 15, 2992 (2015).

252 [5] J. S. Ross, P. Klement, A. M. Jones, N. J. Ghimire, J. Yan, D. G. Mandrus, T. 253 Taniguchi, K. Watanabe, K. Kitamura, W. Yao, D. H. Cobden, and X. Xu, Nat. 254 Nanotechnol. 9, 268 (2014).

255 [6] F. Withers, O. D. Pozo-Zamudio, A. Mishchenko, A. P. Rooney, A. Gholinia, K. 256 Watanabe, T. Taniguchi, S. J. Haigh, A. K. Geim, A. I. Tartakovskii, and K. S. 257 Novoselov, Nat. Mater. 14, 301 (2015).

258 [7] D. Li, R. Cheng, H. Zhou, C. Wang, A. Yin, Y. Chen, N. O. Weiss, Y. Huang, and 259 X. Duan, Nat. Commun. 6. 7509 (2015).

260 [8] F. Withers, O. D. Pozo-Zamudio, S. Schwarz, S. Dufferwiel, P. M. Walker, T. 261 Godde, A. P. Rooney, A. Gholinia, C. R. Woods, P. Blake, S. J. Haigh, K. Watanabe, T. 262 Taniguchi, I. L. Aleiner, A. K. Geim, V. I. Fal'ko, A. I. Tartakovskii, and K. S. 263 Novoselov, Nano Lett. 15, 8223 (2015).

264 [9] A. Pospischil, M. M. Furchi, and M. Mueller, Nat. Nanotechnol. 9, 257 (2014).

265 [10] M. Bernardi, M. Palummo, and J. C. Grossman, Nano Lett. 13, 3664 (2013).

266 [11] B. W. H. Baugher, H. O. H. Churchill, Y. Yang, and P. Jarillo-Herrero, Nat. 267 Nanotechnol. 9, 262 (2014). 
[12] M. Buscema, G. A. Steele, H. S. J. van der Zant, and A. Castellanos-Gomez, Nano

269

270

271

Res. 7, 561 (2014).

[13] S. Tongay, J. Zhou, C. Ataca, J. Liu, J. S. Kang, T. S. Matthews, L.You, J. Li, J. C. Grossman, and J. Wu, Nano Lett. 13, 2831 (2013).

[14] Y. Lin, X. Ling, L. Yu, S. Huang, A. L. Hsu, Y.-H. Lee, J. Kong, M. S. Dresselhaus, and T. Palacios, Nano Lett. 14, 5569 (2014).

[15] K. Kheng, R. T. Cox, Y. Merle d'Aubigné, F. Bassani, K. Saminadayar, and S. Tatarenko, Phys. Rev. Lett. 71, 1752 (1993).

[16] G. Finkelstein, H. Shtrikman, and I. Bar-Joseph, Phys. Rev. B 53, R1709 (1996).

[17] Q. Fu, D. Lee, A. Mysyrowicz, A. V. Nurmikko, R.L. Gunshor, and L. A. Kolodziejski, Phys. Rev. B 37, 8791 (1988).

[18] R. Cingolani, Y. Chen, and K. Ploog, Phys. Rev. B 38, 13478 (1988).

[19] A. Singh, G. Moody, S. Wu, Y. Wu, N.J. Ghimire, J. Yan, D.G. Mandrus, X. Xu, and X. Li, Phys. Rev. Lett. 112, 216804 (2014).

[20] A. Suna, Phys. Rev. B 1, 1716 (1970).

[21] L. Lüer, S. Hoseinkhani, D. Polli, J. Crochet, T. Hertel, and G. Lanzani, Nat. Phys. 5, 54 (2009).

[22] N. Kumar, Q. Cui, F. Ceballos, D. He, Y. Wang, and H. Zhao, Phys. Rev. B 89, 125427 (2014).

[23] S. Mouri, Y. Miyauchi, M. Toh, W. Zhao, G. Eda, and K. Matsuda, Phys. Rev. B 90, 155449 (2014).

[24] S. Konabe and S. Okada, Phsy. Rev. B 90, 155304 (2014).

[25] L. Yuan and L. Huang, Nanoscale 7, 7402 (2015).

[26] Y. Yu, Y. Yu, C. Xu, A. Barrette, K. Gundogdu, and L. Cao, Phys. Rev. B 93, 201111 (2016). 
[27] A. Surrente, A. A. Mitioglu, K. Galkowski, L. Klopotowski, W. Tabis, B. Vignolle,

294

295

296

297

298

299

300

301

302

303

304

305

306

307

308

309

310

311

312

313

314

315

316

317
D. K. Maude, and P. Plochocka, Phys. Rev. B 94, 075425 (2016).

[28] C. Robert, R. Picard, D. Lagarde, G. Wang, J. P. Echeverry, F. Cadiz, P. Renucci, A. Högele, T. Amand, X. Marie, I. C. Gerber, and B. Urbaszek, Phys. Rev. B 94, $155425(2016)$.

[29] G. Froehlicher, E. Lorchat, and S. Berciaud, Phys. Rev. B 94, 085429 (2016).

[30] R. A. Taylor, R. A. Adams, J. F. Ryan, R. M. Park, J. Cryst. Growth 159, 822 (1996).

[31] X. Cui, G.-H. Lee, Y.D. Kim, G. Arefe, P. Y. Huang, C.-H. Lee, D. A. Chenet, X. Zhang, L. Wang, F. Ye, F. Pizzocchero, B. S. Jessen, K. Watanabe, T. Taniguchi, D. A. Muller, T. Low, P. Kim, and J. Hone, Nat. Nanotechnol. 10, 534 (2015).

[32] S. Behura, P. Nguyen, S. Che, R. Debbarma, and V. Berry, J. Am. Chem. Soc. 137, $13060(2015)$.

[33] F. Cadiz, E. Courtade, C. Robert, G. Wang, Y. Shen, H. Cai, T. Taniguchi, K. Watanabe, H. Carrere, D. Lagarde, M. Manca, T. Amand, P. Renucci, S. Tongay, X. Marie, and B. Urbaszek, arXiv:1702.00323v1 [cond-mat.mtrl-sci] (unpublished).

[34] N. Peimyoo, J. Shang, C. Cong, X. Shen, X. Wu, E. K. L. Yeow, and T. Yu, ACS Nano 12, 10985 (2013).

[35] X.-X. Zhang, Y. You, S.Y.F. Zhao, and T.F. Heinz, Phys. Rev. Lett. 115, 257403 (2015).

[36] H. R. Gutiérrez, N. Perea-López, A. L. Elías, A. Berkdemir, B. Wang, R. Lv, F. López-Urías, V. H. Crespi, H. Terrones, and M. Terrones, Nano Lett. 13, 3447 (2013).

[37] K. Watanabe, T. Taniguchi, and H. Kanda, Nat. Mater. 3, 404 (2004).

[38] M. Okada, T. Sawazaki, K. Watanabe, T. Taniguchi, H. Hibino, H. Shinohara, and R. Kitaura, ACS Nano 8, 8273 (2014). 
[40] S. Mouri, Y. Miyauchi, and K. Matsuda, Nano Lett. 13, 5944 (2013).

[41] Y. Li, A. Chernikov, X. Zhang, A. Rigosi, H. M. Hill, A. M. Zande, D. A. Chenet,

[42] See Supplemental Material at [ url ] for the IRF signal and the exciton PL decay signal, PL decay signals at various initial exciton densities, and the time-integrated exciton density plotted as a function of the EEA rate constant.

[43] P. E. Shaw, A. Ruseckas, and I. D. W. Samuel, Adv. Mater. 20, 3516 (2008).

[44] C. R. Dean, A. F. Young, I. Meric, C. Lee, L. Wang, S. Sorgenfrei, K. Watanabe, T. Taniguchi, P. Kim, K. L. Shepard, and J. Hone, Nat. Nanotechnol. 5, 722 (2010). [45] A.V. Stier, N.P. Wilson, G. Clark, X. Xu, and S.A. Crooker, Nano Lett.16, 7054 (2016).

[46] G. Bastard, E. E. Mendez, L. L. Chang, and L. Esaki, Phys. Rev. B 26, 1974 (1982).

[47] P. J. Caplan, E. H. Poindexter, B. E. Deal, and R. R. Razouk, J. Appl. Phys. 50, 5847 (1979).

[48] H. Hillmer, A. Forchel, R. Sauer, and C. W. Tu, Phys. Rev. B 42, 3220 (1990).

[49] M. Palummo, M. Bernardi, and J. C. Grossman, Nano Lett. 15, 2794 (2015).

[50] M. Amani, P. Taheri, R. Addou, G. H. Ahn, D. Kiriya, D.-H. Lien, J. W. Ager III, R. M. Wallace, and A. Javey, Nano Lett. 16, 2786 (2016). 
342 FIG. 1. (Color online) Optical microscopy images and schematic structures of (a) $343 \mathrm{WS}_{2} / \mathrm{hBN}$ and (b) $\mathrm{WS}_{2} / \mathrm{SiO}_{2}$ heterostructured samples. White broken lines in (a) depict $3441 \mathrm{~L}-\mathrm{WS}_{2}$ regions. The scale bar is $5 \mu \mathrm{m}$ long. Steady-state PL spectra of $\mathrm{WS}_{2} / \mathrm{hBN}$ (red)

FIG. 2. (Color online) Normalized exciton PL decay signals for (a) $\mathrm{WS}_{2} / \mathrm{hBN}$ and (b) $\mathrm{WS}_{2} / \mathrm{SiO}_{2}$ at different initial exciton densities. Instrumental response function (IRF) signals are shown for reference as dashed lines. (c) Average decay times as functions of initial exciton densities for $\mathrm{WS}_{2} / \mathrm{hBN}$ (filled circles) and $\mathrm{WS}_{2} / \mathrm{SiO}_{2}$ (open circles). double-exponent fitting of the measured decay signal.

FIG. 3. (Color online) Data expressed in the linearized form of Eqn. (2) for (a) $\mathrm{WS}_{2} / \mathrm{hBN}$ and (b) $\mathrm{WS}_{2} / \mathrm{SiO}_{2}$ heterostructures, with $n_{0}$ denoting the initial exciton density and lines representing linear fits to the above data. (c) The slope $\left(1 / n_{0}+\gamma \tau\right)$ of linear fits as a function of reciprocal initial exciton density for $\mathrm{WS}_{2} / \mathrm{hBN}$ (filled circles) and $\mathrm{WS}_{2} / \mathrm{SiO}_{2}$ (open circles). Inset shows the zoomed-in region for $\mathrm{WS}_{2} / \mathrm{hBN}$. Decay curves derived from Eqn. (2) at various initial exciton densities for (d) $\mathrm{WS}_{2} / \mathrm{hBN}$ and (e) $\mathrm{WS}_{2} / \mathrm{SiO}_{2}$. Filled circles represent experimental results, solid lines denote decay curves 

$\left.n_{0} \times \exp (t / \tau)\right)$

368

Table 1 EEA rate constant of the $\mathrm{WS}_{2} / \mathrm{hBN}$ and $\mathrm{WS}_{2} / \mathrm{SiO}_{2}$ samples given in this study. 
(a) $\quad \mathrm{WS}_{2} / \mathrm{hBN}$
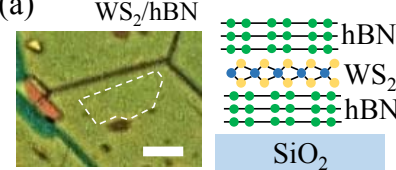

377

378

379

380

381

(c)

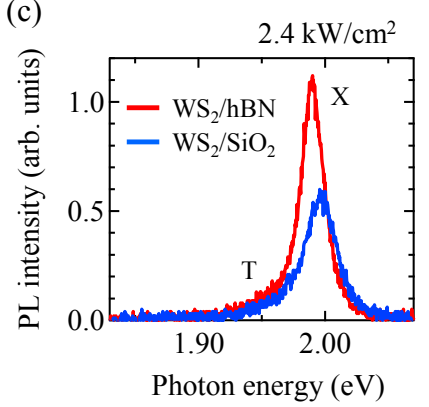

(b)

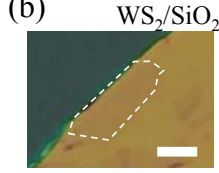

(d)

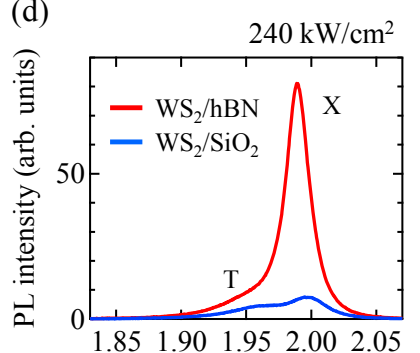

Photon energy (eV)

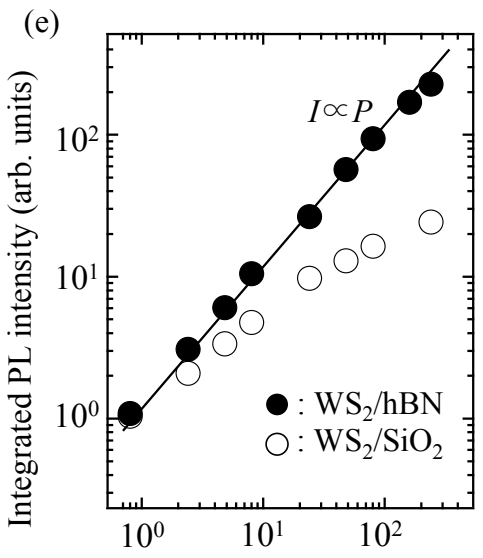

Excitation power density $\left(\mathrm{kW} / \mathrm{cm}^{2}\right)$

382

383

384

385

386

387 


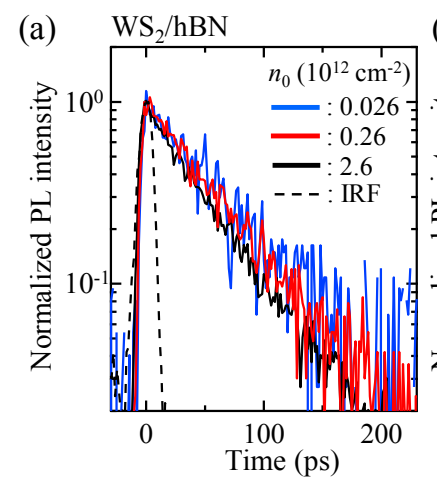

(b) $\quad \mathrm{WS}_{2} / \mathrm{SiO}_{2}$

(c)

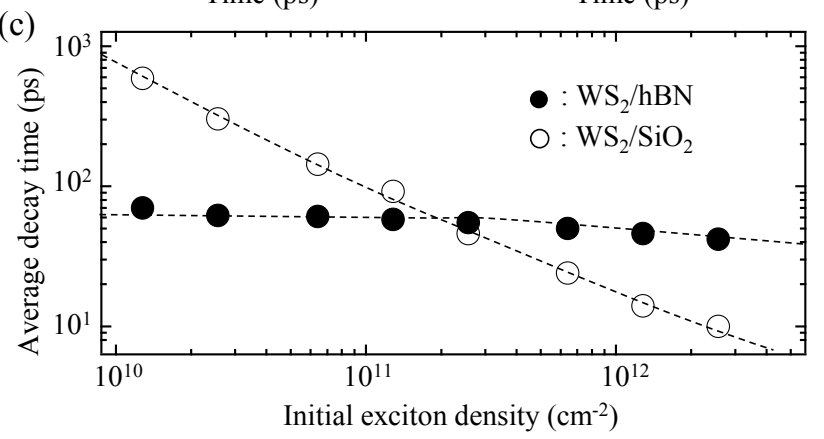

401 
403

404

(a)

405

406

407

408

409

410

411

412

413

414

415 (c)

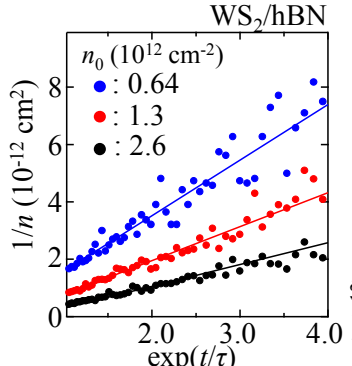

(b)

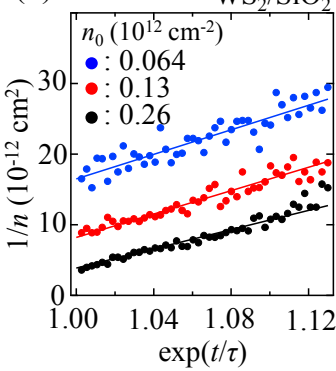

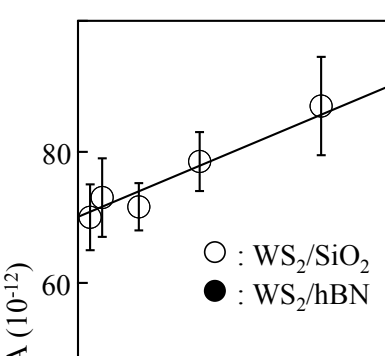

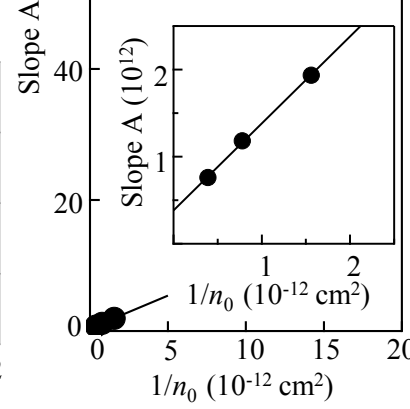

(d)
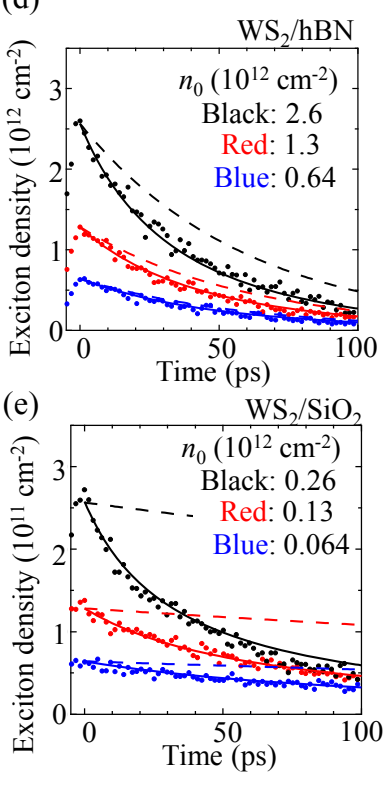


\begin{tabular}{|c|c|c|}
\hline & $\mathrm{WS}_{2} / \mathrm{hBN}$ & $\mathrm{WS}_{2} / \mathrm{SiO}_{2}$ \\
\hline $\begin{array}{c}\text { EEA rate constant } \\
\left(\mathrm{cm}^{2} \mathrm{~s}^{-1}\right)\end{array}$ & $(6.3 \pm 1.7) \times 10^{-3}$ & $(1.2 \pm 0.1) \times 10^{-1}$ \\
\hline
\end{tabular}

418

419

420 